\title{
Current Management of Chronic Kidney Disease: Literature Review
}

\author{
Belayneh Kefale* \\ Department of Pharmacy, Ambo University, Ethiopia
}

Submission: August 07, 2018; Published: December 04, 2018

*Corresponding author: Belayneh Kefale, Clinical pharmacy research team, Department of Pharmacy, College of Medicine and Health Science, Ambo University, Ambo, Ethiopia

Abstract

Chronic kidney disease is a progressive, irreversible decline in renal function in which the body's ability to maintain metabolic and fluid and electrolyte balance fails, resulting in uremia or azotemia. It is not a single disease and associated with different medical conditions such as diabetes, hypertension and anemia; Even though it is caused by primary kidney disease (e.g., glomerular diseases, tubulointerstitial diseases, obstruction, and polycystic kidney disease). Early detection, prevention, evaluation, and management of chronic kidney disease and antecedent conditions could prevent complications of decreased kidney function, slow the progression of kidney disease to kidney failure, and reduce cardiovascular disease risk.

Most patients with chronic kidney disease will die of events related to cardiovascular disease before end-stage renal disease develops. Different studies used as evidence that mild to moderate degrees of renal impairment are associated with increased cardiovascular risk. Therefore; an important focus of care for patients with chronic kidney disease includes management of cardiovascular risk factors and other complications and life style modification. There is no cure for kidney disease, but it may be possible to discontinue its progress or at least slow down the progress. At last dialysis and transplant are the most frequent treatment methods for end stage renal failure. But these treatment methods are not affordable by most of the population. This makes the development of new therapeutic options for treating chronic kidney disease increasingly important.

Keywords: Chronic renal failure; Chronic kidney disease complication; Renal failure management, Haemodialysis; Transplantation.

Abbrevations: ACE: Angiotensin-Converting Enzyme; ARB: Angiotensin-Receptor Blocker; CAKUT: Kidney and urinary tract; CVD: Cardiovascular disease; CKD: Chronic Kidney Disease; CRF: Chronic Renal Failure; eGFR: Estimated glomerular filtration rate; ESRD: End-stage renal disease; GFR: Glomerular Filtration Rate; K/DOQI: Kidney Dialysis Outcomes and Quality Initiative; LDL: Low-Density Lipoprotein; LVH: Left ventricular hypertrophy; RAS: Renin- Angiotensin-System

\section{Introduction}

Chronic Renal Failure (CRF) is a global public health crisis that tends to take dimensions of epidemic and has severe impact on quality of patient's life [1]. It is a progressive, irreversible deterioration in renal function in which the body's ability to sustain metabolic and fluid and electrolyte balance fails, resulting in uremia or azotemia (retention of urea and other nitrogenous wastes in the blood) [2]. The kidneys regulate the composition and volume of blood, remove metabolic wastes in the urine, and help control the acid/ base balance in the body. It is typically a progressive disease and is defined as; reduction of kidney functiondefined as an estimated glomerular filtration rate (eGFR) $<60$ $\mathrm{mL} / \mathrm{min} / 1.73 \mathrm{~m}^{2}$ and/ or evidence of kidney damage, including persistent albuminuria-defined as $>30 \mathrm{mg}$ of urine albumin per gram of urine creatinine. It is virtually always asymptomatic in its early stages $[3,4]$.
It is not a single disease and defined based on the presence or absence of kidney damage and level of kidney function irrespective of the type of kidney disease. It is categorized by the level of kidney function, based on GFR, into stages 1 to 5, with each increasing number indicating a more advanced stage of the disease, as defined by a declining GFR (Table 1). This classification system from the National Kidney Foundation's Kidney Dialysis Outcomes and Quality Initiative (K/DOQI) also accounts for structural evidence of kidney injure [5]. There is a mixture of causes and the damage is usually irreversible and can lead to ill health. The main risk factors, which lead to chronic renal failure, are diabetes, hypertension, anemia, osteodystrophy, glomerulonephritis, malnutrition and polycystic kidney disease $[1,6]$. Decisions regarding risk factor modification should be taken on an individual basis. In some cases, dialysis or transplantation may become necessary. 
Table 1: Stages of chronic kidney disease based on kidney function estimated by glomerular filtration rate [3].

\begin{tabular}{|c|c|c|c|}
\hline \multirow{2}{*}{ Stages } & Description & GFR & \multirow{2}{*}{ Related terms } \\
\cline { 2 - 3 } & Kidney damage with normal or $\uparrow$ GFR & ml/min $/ \mathbf{1 . 7 3 \mathbf { m } ^ { 2 }}$ \\
\hline 2 & Kidney damage with mild $\downarrow$ GFR & a & albuminuria, proteinuria, hematuria \\
\hline 3 & Moderate $\downarrow$ GFR & $30-59$ & albuminuria, proteinuria, hematuria \\
\hline 4 & Severe $\downarrow$ GFR & $15-29$ & chronic renal insufficiency, early renal insufficiency \\
\hline 5 & Kidney failure & $<15$ (or dialysis) & renal failure, uremia, end stage, renal insufficiency, late renal insufficiency, pre-ESRD \\
\hline
\end{tabular}

Owing to recent advances in cardiovascular management, chronic renal failure has become a much less frequent complication than in the past; renal failure itself is no longer life-threatening because hemodialysis is available [7]. Hemodialysis and transplant are the most frequent treatment methods for CRF. However, it has been argued that several restrictions and modifications accompany this treatment, which have a detrimental impact on the quality of patient's life and affect individuals' physical and psychological well-being [6]. Thus, the enormous costs of chronic renal failure to the society at large make management of CRF a critical public health priority.

CKD has a complicated interrelationship with other diseases [8]. It is a major risk factor for increased cardiovascular disease and death. Recent studies have reported that CKD is an independent risk factor for cardiovascular disease (CVD) [9]. Therefore, kidney dysfunction should be an additional target for intervention and prevention of CVD $[10,11]$. Due to the asymptomatic nature of this disease, CKD is not frequently detected until its later progress, resulting in lost opportunities for prevention. Progress to kidney failure or other adverse outcomes could be prevented or delayed through early detection and treatment of CKD [12]. Serum creatinine concentration is the most commonly used biomarker to predict the level of kidney function, but it can be affected by various factors such as age, gender, ethnicity, muscle mass, dietary habit and specific drug use.

\section{Chronic Kidney Disease-Related Complications}

Most patients with CKD will die of events related to cardiovascular disease before ESRD develops [13,14]. Therefore, an important focus of care for patients with CKD includes management of cardiovascular risk factors. Progression of CKD is associated with a number of serious complications and the potential complications of chronic renal failure that concern the health professional and that necessitate a collaborative approach to care include; hyperkalemia (due to decreased excretion, metabolic acidosis, catabolism) and excessive intake (diet, medications, fluids); pericarditis, pericardial effusion, and pericardial tamponade due to retention of uremic waste products and inadequate dialysis; hypertension due to sodium and water retention and malfunction of the renin-angiotensin-aldosterone system [15].

Anemiaisoccurredduetodecreased erythropoietinproduction, decreased red blood cell life span, bleeding in the gastrointestinal tract from irritating toxins and ulcer formation, and blood loss during hemodialysis [16]. The anemia of CKD increases morbidity and mortality from cardiovascular complications (angina, left ventricular hypertrophy (LVH) and worsening heart failure) [17], which may lead to further deterioration of renal function and the establishment of a vicious cycle termed the "cardiorenal anemia syndrome". Transfusion is considered as the mainstay of treatment and improved the survival of anemic CKD patients. Bone disease and metastatic and vascular calcifications are due to retention of phosphorus, low serum calcium levels, abnormal vitamin D metabolism, and elevated aluminum levels. Diabetes is associated with adverse outcomes in all stages of CKD [18]. Dyslipidemia is a major risk factor for cardiovascular morbidity and mortality and is common among patients with CKD. The prevalence of hyperlipidemia increases as renal function declines, with the degree of hypertriglyceridemia and elevation of LDL cholesterol being proportional to the severity of renal impairment. Studies also suggest that hyperparathyroidism and the accumulation of calcium in pancreatic islet cells likely contribute to dyslipidemia of CKD as well [19]. Several cardiovascular risk factors associated with CKD are unique to patients with this disease.

\section{Management of chronic kidney Disease and its complication}

The goal of management is to maintain kidney function and homeostasis by treating any underlying condition, slow down the progression, reduce the risk of developing cardiovascular disease (CVD) for as long as possible [10]. In early stages of chronic renal disease; there are usually no symptoms to indicate the kidneys are damaged because kidneys do a good job even when they are not functioning at 100 percent. There is no cure for kidney disease, but it may be possible to discontinue its progress or at least slow down the damage. In many cases, the acceptable treatment 
and lifestyle changes can help keep a person and their kidneys healthier longer. In addition to eating right and taking prescribed medicines, exercising regularly and not smoking are helpful to prolonging kidney health [20].

The management of CKD has focused on diagnosis and treatment of specific kidney diseases, and dialysis or transplantation for kidney failure. Evidence suggests that preventive strategies could substantially reduce the CKD burden, and there is evidence that such strategies are not yet in place. Tests to detect kidney disease at the earliest, most treatable stages are not widely used. Thus, a comprehensive public health approach to address CKD prevention is needed to effectively address this major public health problem [21].

Attention to cardiovascular risk factors remains the cornerstone of care to delay progression of CKD and prevent cardiovascular events. The direct management of CKD focuses on renin angiotensin aldosterone blockade (RAAS) and blood pressure control. Management also includes optimal management of common comorbid conditions such as diabetes and addressing cardiovascular risk factors to decrease risk for CVD [11].

\section{Management of Chronic Kidney Disease with Hypertension}

Hypertension is commonly associated with CKD. It develops in more than $75 \%$ of patients with CKD at any stage [22]. It is both a cause and a consequence of CKD. Given the renal protective effects of angiotensin-converting enzyme (ACE) inhibitors or angiotensin receptor blockers, this class of agents are optimal first-line agents in patients with proteinuria ( $>1 \mathrm{gm} / 24 \mathrm{~h})$, progressive diabetic and nondiabetic renal disease. Angiotensin causes greater vasoconstriction of efferent arteriole than afferent arteriole, leading to glomerular hypertension. This leads to hyperfiltration and prolonged hyperfiltration, leading to glomerular structural and functional deterioration. Both ACEI and ARB can reverse this process and delay renal disease progression. While the reduction of intraglomerular pressure has long-term benefit, it may cause a small rise in serum creatinine in the short term, since GFR is directly correlated to intraglomerular pressure. A rise of up to 20 $30 \%$ above the baseline is acceptable and not a reason to withhold treatment unless hyperkalemia develops [23].

Checking serum creatinine and potassium about 1-2 weeks after initiating or changing the dose of ACEI or ARB is recommended. ACEIs and ARBs do not differ significantly in terms of overall mortality, progression to ESRD, or their antiproteinuria effects. Initial selection of a specific drug should be based on cost, potential side effects, and patient preference. Both classes of medications have been studied extensively. However, a higher volume of evidence and more landmark studies have been done with ACEIs than with ARBs. However, ACEIs have a higher rate of cough and may cause a slightly greater increase of potassium and serum creatinine levels compared with ARBs. With decreasing kidney function, starting doses for both ACEIs and ARBs are lower. Dose titration should occur slowly as needed for control of blood pressure or albuminuria. When starting an ACEI or an ARB, monitoring blood pressure, potassium, and serum creatinine levels is important. Potassium and/or serum creatinine are expected to increase when starting or changing the dose of an ACEI or an ARB. The medication may need to be reduced or discontinued if the potassium level remains elevated at $>5.5 \mathrm{mEq} / \mathrm{L}$ or if the serum creatinine continues to rise $(30 \%$ from baseline) or does not improve [24].

Several large RCTs are currently ongoing to assess the role of dual therapy for CKD patients specifically. Evidence from different studies dual therapy with ACEI and ARB is not recommended and no significant benefit over mono therapy rather increased the risk of worsening kidney function and hyperkalemia [25] (Table 2).

Table 2: Targets of blood pressure in Chronic Kidney Disease [22].

\begin{tabular}{|c|c|}
\hline Urine Albumin Excretion & Blood Pressure Goal \\
\hline$<30 \mathrm{mg} / 24$ hours & $<140 / 90$ (recommended) \\
\hline$>30 \mathrm{mg} / 24$ hours & $<130 / 80$ (suggested) \\
\hline
\end{tabular}

The combination of ACEI and spironolactone is commonly seen in patients with concomitant heart failure but may also be considered for those with severe albuminuria with nephrology input. Patients on combined spironolactone and ACEI or ARB therapy should be monitored carefully for hyperkalemia [22]. If ACEI or ARB is not effective on its own to control BP, then a thiazide or calcium channel blocker may be added. It should be noted that calcium channel blockers should not be prescribed without the concomitant usage of ACEI or ARB, since their sole use may lead to greater hyperfiltration and albuminuria. Once GFR declines to stage G4 or worse, thiazides are generally ineffective, and loop diuretics (e.g., furosemide) are usually needed to control volumedependent hypertension [22]. It should also be noted that patients with more advanced CKD often have resistant hypertension requiring multiple medications [26].

\section{Management of Chronic Kidney Disease with Diabetes Miletus}

Diabetes is associated with adverse outcomes in all stages of CKD. Patients with diabetes are at increased risk for CKD and cardiovascular events. Thus, it is important for clinicians to appreciate the importance of glycemic control for CKDDM patients [27]. Relatively strict control of blood glucose (hemoglobin $\mathrm{A} 1 \mathrm{C} \leq 7 \%$ ) in both type 1 and type 2 diabetes reduces the development of diabetic nephropathy and its progression. Diligent BP control reduces renal disease progression and cardiovascular morbidity and mortality among patients with diabetes [25]. Dose adjustments based on renal function are noted. Renal deterioration leads to decreased renal metabolism of hypoglycemic drugs and/or insulin. As a result, dose adjustment of these medications may be required as CKD progresses to prevent hypoglycemia [4]. Metformin is an inexpensive and 
effective oral hypoglycemic agent that is recommended as firstline therapy for both overweight and non-overweight patients with type 2 diabetes mellitus. There is much concern about the safety of metformin in CKD (stage 3-5), particularly the risk for lactic acidosis (Table 3) [28]. Insulin and thiazolidinediones are suitable for patients with renal impairment. Studies showed that as the CKD stage increased, the use of insulin also increased, but the use of thiazolidinediones remained constant [27].

Table 3: Suggestions for the Management of Cardiovascular Risk Factors in Patients with Advanced Chronic Kidney Disease [33].

\begin{tabular}{|c|c|}
\hline Risk Factor & Suggested Management \\
\hline Smoking & Recommend smoking cessation \\
\hline Diet & Recommend sodium intake of $<2.4 \mathrm{~g}$ per day. \\
\hline Weight & Recommend maintaining body-mass index at $<25$ and waist circumference at $<102 \mathrm{~cm}$ for men and $<88 \mathrm{~cm}$ for women. \\
\hline Exercise & $\begin{array}{l}\text { For patients for whom it is feasible, recommend 30-60 min of moderate-intensity dynamic exercise (eg walking, jogging, } \\
\text { cycling, or swimming) 4-7 days per week. }\end{array}$ \\
\hline Hypertension & $\begin{array}{c}\text { Target blood pressure should be }<130 / 80 \mathrm{~mm} \text { Hg; patients with chronic kidney disease but without proteinuria (ratio of } \\
\text { albumin [measured in milligrams per deciliter] to creatinine [measured in milligrams] of }<0.3 \text { ) should be treated with an ACE } \\
\text { inhibitor, an ARB, a loop diuretic, a beta blocker (in patients younger than } 60 \text { years), a calcium-channel blocker, or some combi- } \\
\text { nation of these drugs. }\end{array}$ \\
\hline Proteinuria & $\begin{array}{l}\text { Patients with chronic kidney disease and proteinuria (ratio of albumin [measured in milligrams per deciliter] to creatinine } \\
\text { [measured in milligrams] of }>0.3 \text { ) should be treated with an ACE inhibitor or an ARB. }\end{array}$ \\
\hline Diabetes mellitus & $\begin{array}{l}\text { Target glycated hemoglobin level should be }<7.0 \% \text { and target fasting plasma glucose level, } 90-160 \mathrm{mg} / \mathrm{dl} \text { ( } 5.0-8.9 \mathrm{mmol} / \mathrm{liter}) \\
\text { treatment with metformin is acceptable in patients with stable stage I, II, or III chronic kidney disease; short-acting sulfony- } \\
\text { lureas (e.g., gliclazide) are preferable to long-acting agents; sulfonylureas and insulin require dose adjustment; repaglinide can } \\
\text { be used in patients with stage IV chronic kidney disease and needs no dose adjustment. }\end{array}$ \\
\hline Dyslipidemia & $\begin{array}{l}\text { Targets for LDL cholesterol levels should follow guidelines for the general population; statin therapy is recommended; no dose } \\
\text { adjustment is required for bile acid sequestrants, statins, niacin, or ezetimibe, but fibrates require dose adjustment according } \\
\text { to their effect on kidney function. }\end{array}$ \\
\hline Anemia & $\begin{array}{l}\text { Iron supplementation is recommended; erythropoiesis-stimulating agents have been used but caution is recommended } \\
\text { because of cardiovascular risks associated with the use of these agents; target hemoglobin level should be no higher than } 10 \text { to } \\
\qquad 12 \mathrm{mg} / \mathrm{dl} \text { if erythropoiesis-stimulating agents are being used. }\end{array}$ \\
\hline Other & $\begin{array}{l}\text { Aspirin, at a dose of } 81 \mathrm{mg} \text { daily, is recommended if cardiovascular risk is high or cardiovascular disease is present and if there } \\
\text { is no contraindication to aspirin. }\end{array}$ \\
\hline
\end{tabular}

\section{Management of Chronic Kidney Disease with Cardiovascular disease}

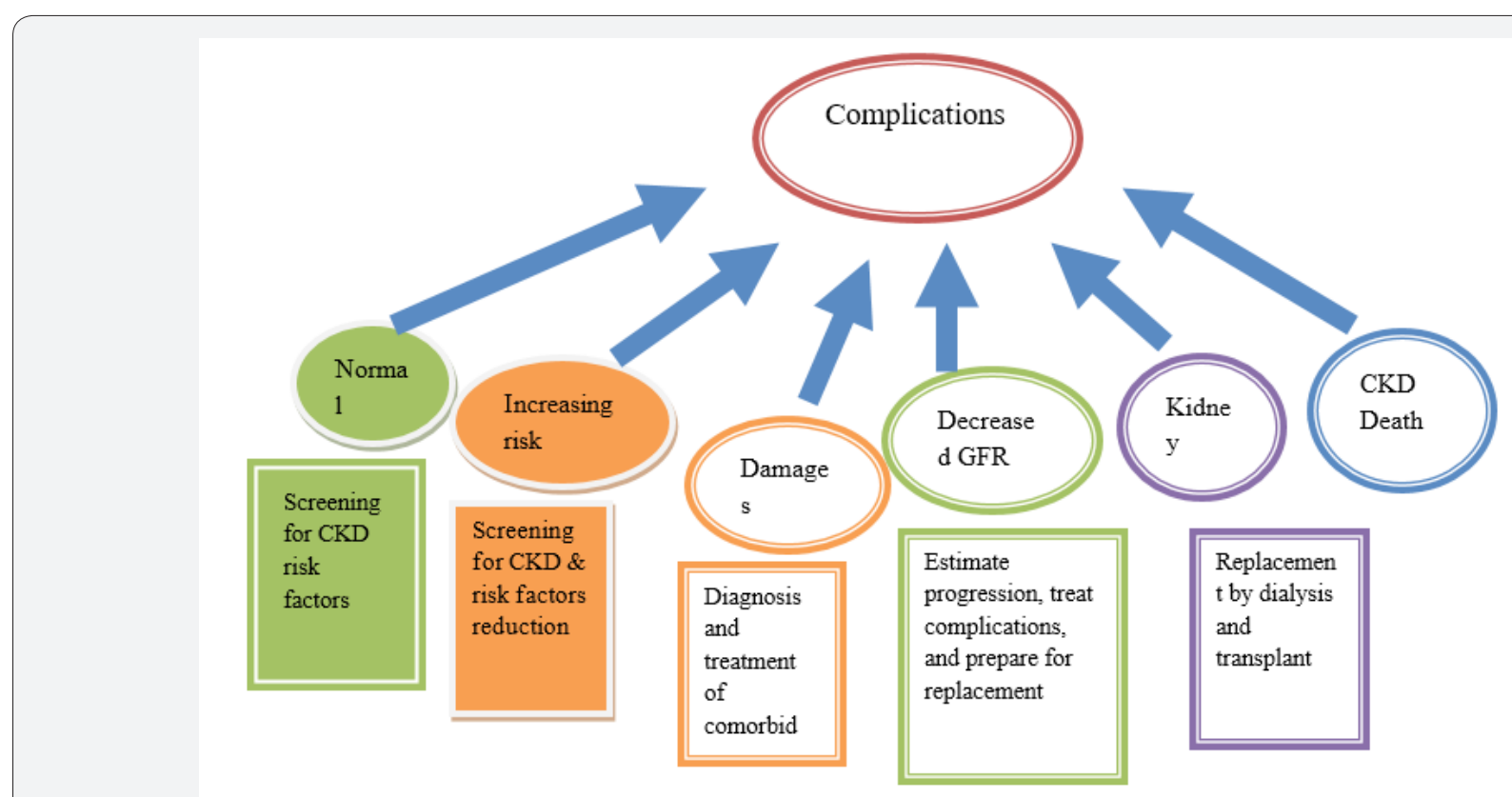

Figure 1: Stage of chronic kidney disease and its therapeutic intervention: A new framework [13]. 
Cardiovascular disease is the cause of morbidity and mortality among patients with CKD. CKD patients have a risk of cardiovascular disease (CVD) that is 10 to 30 times that of people without kidney disease [23]. Recent studies have demonstrated that even early stage CKD constitutes a significant risk factor for cardiovascular events and death. Similarly, CVD is a risk factor for progression of CKD. The frequency of cardiovascular complications in patients with CKD can be reduced with appropriate treatment of other CVD risk factors (10). There is a high prevalence of dyslipidemia among patients at every stage of chronic kidney disease [29]. Thus, screening, evaluation and therapeutic interventions for control of dyslipidemia are important (Figure 1).

Statins is used to reduce the relative risk of cardiovascular events to a similar extent among patients with and without CKD (Table 3). However, the benefit is greater in patients with CKD because of the greater baseline risk for patients with CKD. In addition to reducing cardiovascular risk, statins may also have a role in preventing progression of kidney disease and reducing albuminuria, though evidence for these outcomes is less robust [30].

\section{Management of Chronic Kidney Disease with other complications}

The anemia of CKD is treated via recombinant human erythropoietin [31]. This intervention has replaced transfusions as the mainstay of treatment and improved the survival of anemic CKD patients (Table 3) [17]. In treatment of mineral metabolism abnormalities in patients with CKD dietary phosphate restriction should be used continuously to treat hyperphosphatemia. Therapy with calcium-containing phosphate binders (calcium carbonate or calcium acetate) should be initiated if dietary restriction fails to control hyperphosphatemia and if hypercalcemia is not present (If hypercalcemia develops the dose of calcium-containing phosphate binders or vitamin D analogues should be reduced). Consider prescribing vitamin-D analogues if serum levels of intact parathyroid hormone are $>53 \mathrm{pmol} / \mathrm{L}$. Therapy should be discontinued if hypercalcemia or hyperphosphatemia develops or if parathyroid hormone levels are $<10.6 \mathrm{pmol} / \mathrm{L}$. Vitamin-D analogues should be used in conjunction with a specialist with experience in prescribing these agents. There is insufficient evidence to recommend use of phosphate binders that do not contain calcium, novel vitamin-D analogues or calcimimetics [22].

In water and electrolyte balance; Precise restriction of fluid intake is only required for patients with oliguric end-stage kidney disease. Patients should avoid binge drinking and be vigilant in replacing extra fluid losses in hot weather and during episodes of diarrhoea or vomiting. Severe acute volume overload may require high-dose loop diuretics or dialysis [26].

If the potassium level rises above $7 \mathrm{mmol} / \mathrm{L}$, it is treated with dialysis. Otherwise, treatment is directed towards the cause - eg, excess fruit, chocolate or coffee, gastrointestinal haemorrhage, acidosis or tissue necrosis [32]. Chronic acidosis aggravates hyperkalaemia, inhibits protein synthesis and accelerates calcium loss from bone. Regarding oral bicarbonate supplements in the management of metabolic acidosis: consider oral sodium bicarbonate supplementation for people with a GFR less than 30 $\mathrm{ml} /$ minute $/ 1.73 \mathrm{~m}^{2}$, and a serum bicarbonate concentration of less than $20 \mathrm{mmol} / \mathrm{L}$ [33].

\section{Renal Replacement Therapy and Dialysis}

Patients with stage 5 chronic kidney disease have ESRD with a GFR of $15 \mathrm{ml} / \mathrm{min}$ or less. At this advanced stage of kidney disease, the kidneys have lost nearly all their ability to do their job effectively, and eventually dialysis or kidney transplant is needed to live [34]. Because the kidneys are no longer able to remove waste and fluids from the body, toxins build up in the blood, causing an overall ill feeling. Kidneys also will become no longer able to perform such as regulating blood pressure, producing the hormone that helps make red blood cells and activating vitamin D for healthy bones. In dialysis treatment for people with stage 5 CKD, toxins are removed from the blood; medicines replace the functions of the kidney. Kidney transplant is another treatment option in ESRF that is necessary to continue living [20].

\section{Future perspective of CKD management}

The conventional approach of management of CKD includes dialysis and renal transplantation, which are not affordable by most population mainly due to economic reasons [35]. Thus, exploration of a safe and alternative therapy is needed, which proves to be helpful in reducing the requirement of dialysis and in postponing the renal transplantation [36]. During diagnosis current imaging modalities especially ultrasound (US), computed tomography, and magnetic resonance imaging (MRI) provide adequate information on structural changes but little on functional impairment in CKD. Although not yet considered firstline procedures for evaluating patients with renal disease, new US and MR imaging techniques may permit the assessment of renal function in the near future. Recent developments in molecular imaging indicate that pathophysiological pathways of renal diseases such as apoptosis, coagulation, fibrosis, and ischemia will be visualized at the tissue level and imaging is expected to play an increasing role in the management of CKD [37].

CKD is invariably characterized by progressive kidney fibrosis and at present, treatment aiming to slow the progression of CKD is limited to aggressive blood pressure, diabetes and other risk factor control, with few therapies targeting the fibrotic process itself. However, kidney disease can lead to kidney failure (less than $10 \%$ kidney function). Once this happens, patients need dialysis or a kidney transplant to stay alive [36]. But there are not enough organ donors to provide transplants for CKD patients. This makes the development of new therapeutic options for treating CKD increasingly important.

\section{Kidney stem cells}

The most convincing evidence for the existence of stem cells have key features and researchers have shown them to 
be responsible for production of podocytes - specialised cells involved in the filtration work of the nephron and that need to be replaced continuously throughout our lifetime. Studies also suggest that these same proposed stem cells might be able to generate a second type of specialized cell found in the nephron lining, called proximal tubular epithelial cells. Various animal and human studies demonstrated the role of stem cells in repair and regenerations of kidney [38].

\section{Kidney Disease and Mesenchymal Stem Cells}

A number of different types of cells from the bone marrow have been tested in animals and in clinical studies for potential use in kidney disease. Amongst all the cells under investigation, mesenchymal stem cells (MSCs) have shown the most promising results to date. Studies suggest that MSCs may be able to enhance the intrinsic ability of the kidney to repair itself $[38,39]$. Researchers investigating the therapeutic effects of these MSCs within the kidney have suggested these cells may release proteins that can help kidney cells to grow, inhibit cell death and that could encourage the kidney's own stem cells to repair kidney damage [40]. Further research is needed to establish whether these ideas are correct and if so, how this could lead to a treatment for patients [39]. More research is needed to identify their precise role in normal kidney maintenance and to investigate their potential to enhance the kidney's ability to regenerate or repair itself after damage.

Another type of stem cell that scientists are using in kidney research is the induced pluripotent stem cell (iPSC). iPSCs are made by reprogramming adult, specialized cells of the body to act like embryonic stem cells. They have the ability to develop into any cell or tissue in the body. Induced pluripotent stem (iPS) cell technology, which allows derivation of patient-specific pluripotent stem cells, could provide a possible alternative modality for kidney replacement therapy for patients with ESRD [41]. Recently researchers have been able to use iPSCs to produce kidney cells in a very early stage of development. These very early kidney cells resemble cells found in the embryo that will turn into the cells that eventually make up the kidney in foetal development. These cells could have the potential to make the glomerulus and tubules, the building blocks of the nephron [42]. However, a lot of research needs to be done before such cells can be used in patients to treat CKD.

An organ replacement is also under investigation and may help kidney disease patients in the future: The use of organ scaffolds to produce whole, transplant Table organs. Organ scaffolds are organs from which all the cells have been removed. What remains is the extracellular matrix - the part of the organ that supports its shape. This matrix can be seeded with a patient's own cells, which can be carefully nurtured to grow and multiply to re-cover the scaffold. By using the patient's own cells, the complications of immune rejection that can occur with organ transplantations are drastically reduced. The challenge with this approach is identifying and obtaining the right types of cells to seed the scaffold, especially in organs with complex structures made up of many different cells. IPSCs or the recently identified kidney MSCs could be useful candidate cells for seeding kidney organ scaffolds. Very recently, experiments in rats have shown the feasibility of this approach. Stem cell treatments for kidney disease have not yet been developed. Researchers also continue to explore new ideas using emerging technologies in stem cell research, such as reprogramming cells to change their behavior.

\section{Acknowledgement}

The authors are grateful to all health care professionals, researchers and governments working to prevent different medication related problems in CKD. We are also grateful to all the information resources included in this review article.

\section{References}

1. Gerogianni K, Babatsikou P (2014) Psychological aspects in chronic renal failure. Health science journal 8(2):205-214.

2. Leung DK (2003) Psychosocial aspects in renal patients. Peritoneal dialysis international. 23(Supplement 2): S90-S94.

3. Levey AS, De Jong PE, Coresh J, El Nahas M, Astor BC, et al. (2011) The definition, classification, and prognosis of chronic kidney disease: a KDIGO Controversies Conference report. Kidney international 80(1): $17-28$.

4. Control CFD (2010) Prevention National Chronic Kidney Disease Fact Sheet: general information and national estimates on chronic kidney disease in the United States, 2010. Atlanta, GA: US Department of Health and Human Services (HHS), CDC, USA.

5. Emara S, Alzaylai A (2013) Renal failure in burn patients: a review. Ann Burns Fire Disasters. 26(1):12-15.

6. (2002) K/DOQI clinical practice guidelines for chronic kidney disease: evaluation, classification, and stratification. American journal of kidney diseases: the official journal of the National Kidney Foundation. 39(2 Suppl 1): S1-266.

7. Gerogianni K (2003) Stressors of patients undergoing chronic hemodialysis. Nursing 42(2): 228-246.

8. Snively CS, Gutierrez C (2004) Chronic kidney disease: prevention and treatment of common complications. American family physician. 70(10): 1921-1928.

9. Mann JF, Gerstein HC, Pogue J, Bosch J, Yusuf S (2001) Renal insufficiency as a predictor of cardiovascular outcomes and the impact of ramipril: the HOPE randomized trial. Annals of internal medicine 134(8): 629-636.

10. De Zeeuw D, Hillege HL, De Jong PE (2005) The kidney, a cardiovascular risk marker, and a new target for therapy. Kidney international. 68: S25-S29.

11. Sarnak MJ, Levey AS, Schoolwerth AC, Coresh J, Culleton B, et al. (2003) Kidney disease as a risk factor for development of cardiovascular disease a statement from the American Heart Association Councils on kidney in cardiovascular disease, high blood pressure research, clinical cardiology, and epidemiology and prevention. Hypertension. 42(5): 1050-1065.

12. Locatelli F, Del Vecchio L, Pozzoni P (2002) The importance of early detection of chronic kidney disease. Nephrology Dialysis Transplantation. 17(suppl 11): 2-7. 
13. Levey AS, Coresh J (2012) Chronic kidney disease. Lancet (London, England). 379(9811): 165-180.

14. Keith DS, Nichols GA, Gullion CM, Brown JB, Smith DH (2004) Longitudinal follow-up and outcomes among a population with chronic kidney disease in a large managed care organization. Archives of internal medicine. 164(6): 659-663.

15. Combe C, McCullough KP, Asano Y, Ginsberg N, Maroni BJ, et al. (2004) Kidney disease outcomes quality initiative (K/DOQI) and the dialysis outcomes and practice patterns study (DOPPS): nutrition guidelines, indicators, and practices. American journal of kidney diseases 44(5): 39-46.

16. McClellan W, Aronoff SL, Bolton WK, Hood S, Lorber DL, et al. The prevalence of anemia in patients with chronic kidney disease. Current medical research and opinion 20(9): 1501-1510.

17. Besarab A, Levin A. Defining a renal anemia management period American journal of kidney diseases. 2000;36(6): S13-S23.

18. Tonelli M, Keech A, Shepherd J, Sacks F, Tonkin A, et al. (2005) Effect of pravastatin in people with diabetes and chronic kidney disease. J Am Soc Nephrol 16(12): 3748-3754.

19. Wanner C, Krane V, Marz W, Olschewski M, Mann JF, et al. (2005) Atorvastatin in patients with type 2 diabetes mellitus undergoing hemodialysis. N Engl J Med 353(3): 238-248.

20. Stigant C, Stevens L, Levin A (2003) Nephrology: 4. Strategies for the care of adults with chronic kidney disease. Canadian Medical Association Journal 168(12): 1553-1560.

21. Assadi F (2013) Strategies to reduce the incidence of chronic kidney disease in children: Time for action. J Nephrol 26(1): 41-47.

22. Levin A, Hemmelgarn B, Culleton B, Tobe S, McFarlane P, et al. (2008) Guidelines for the management of chronic kidney disease. Canadian Medical Association Journal 179(11): 1154-1162.

23. Assadi F (2008) Relation of left ventricular hypertrophy to microalbuminuria and C-reactive protein in children and adolescents with essential hypertension. Pediatr Cardiol 29(3): 580-584.

24. Levey AS, Coresh J, Bolton K, Culleton B, Harvey KS, et al. (2002) K/ DOQI clinical practice guidelines for chronic kidney disease. American Journal of Kidney Diseases. 39(2 SUPPL. 1): S1-266.

25. Finch N, Syme H, Elliott J (2016) Risk Factors for Developmen of Chronic Kidney Disease in Cats. Journal of Veterinary Internal Medicine. J Vet Intern Med 30(2): 602-610.

26. Stevens P, O'donoghue D, De Lusignan S, Van Vlymen J, Klebe B, et al. (2007) Chronic kidney disease management in the United Kingdom: NEOERICA project results. Kidney Int 72(1): 92-99.

27. Albers JW, Herman WH, Pop-Busui R, Feldman EL, Martin CL, Cleary PA, et al. (2010) Effect of prior intensive insulin treatment during the Diabetes Control and Complications Trial (DCCT) on periphera neuropathy in type 1 diabetes during the Epidemiology of Diabetes Interventions and Complications (EDIC) Study. Diabetes Care 33(5):
1090-1096.

28. Koro CE, Lee BH, Bowlin SJ (2009) Antidiabetic medication use and prevalence of chronic kidney disease among patients with type 2 diabetes mellitus in the United States. Clinical therapeutics 31(11): 2608-2617.

29. (2002) MRC/BHF Heart Protection Study of cholesterol lowering with simvastatin in 20,536 high-risk individuals: a randomised placebocontrolled trial. Lancet (London, England). Lancet 360(9326): 7-22.

30. Palmer SC, Navaneethan SD, Craig JC, Johnson DD, Perkovic V, et al. (2014) HMG CoA reductase inhibitors (statins) for people with chronic kidney disease not requiring dialysis. Sao Paulo Medical Journal 132(5): 314-315.

31. Fink J, Blahut S, Reddy M, Light P (2001) Use of erythropoietin before the initiation of dialysis and its impact on mortality. American journal of kidney diseases: the official journal of the National Kidney Foundation 37(2): 348-355.

32. Rabar S, Harker M, O’Flynn N, Wierzbicki AS. (2014) Lipid modification and cardiovascular risk assessment for the primary and secondary prevention of cardiovascular disease: summary of updated NICE guidance.

33. Abboud H, Henrich WL (2010) Stage IV chronic kidney disease. New England Journal of Medicine 362(1): 56-65.

34. Oniscu GC, Brown H, Forsythe JL. (2004) How great is the survival advantage of transplantation over dialysis in elderly patients? Nephrology Dialysis Transplantation 19(4): 945-951.

35. Woo KT, Wong KS, Chan CM (2009) Clinical trials of the past decade in the management of chronic kidney disease. Reviews on recent clinical trials 4(3): 159-162.

36. Patel MV, Gupta S, Patel NG (2011) Effects of Ayurvedic treatment on 100 patients of chronic renal failure (other than diabetic nephropathy). Ayu 32(4): 483-846.

37. Stevens LA, Levey AS (2009) Current status and future perspectives for CKD testing. Am J Kidney Dis 53(3): S17-S26.

38. Choi S, Park M, Kim J, Hwang S, Park S (2009) The role of mesenchymal stem cells in the functional improvement of chronic renal failure. Stem Cells Dev 18(3): 521-530.

39. Gatti S, Bruno S, Deregibus MC, Sordi A, Cantaluppi V, et al. (2011) Microvesicles derived from human adult mesenchymal stem cells protect against ischaemia-reperfusion-induced acute and chronic kidney injury. Nephrol Dial Transplant, pp. 1474-1483.

40. Eirin A, Lerman LO (2014) Mesenchymal stem cell treatment for chronic renal failure. Stem Cell Res Ther 5(83): 1186

41. Thatava T, Armstrong AS, De Lamo JG, Edukulla R, Khan YK, et al. (2011) Successful disease-specific induced pluripotent stem cell generation from patients with kidney transplantation. Stem Cell Res Ther 2(6): 48.

42. Freedman BS (2015) Modeling Kidney Disease with iPS Cells. Biomarker insights.10(Suppl 1): 153-169. 
(C) This work is licensed under Creative

CC (i) Commons Attribution 4.0 License

DOI: 10.19080/JOJUN.2018.06.555683
Your next submission with Juniper Publishers will reach you the below assets

- Quality Editorial service

- Swift Peer Review

- Reprints availability

- E-prints Service

- Manuscript Podcast for convenient understanding

- Global attainment for your research

- Manuscript accessibility in different formats ( Pdf, E-pub, Full Text, Audio)

- Unceasing customer service

Track the below URL for one-step submission https://juniperpublishers.com/online-submission.php 